\title{
A Computationally Efficient Unified Approach to the Numerical Analysis of the Sensitivity and Noise of Semiconductor Devices
}

\author{
Giovanni Ghione, Member, IEEE, and Fabio Filicori
}

\begin{abstract}
This paper presents a new computationally efficient unified approach to the numerical simulation of sensitivity and noise in majority-carrier semiconductor devices, based on the extension to device simulation of the adjoint method, well known in the sensitivity and noise analysis of electrical networks. Sensitivity and device noise analysis based on physical models are shown to have a common background, since they amount to evaluating the small-signal device response to an impressed, distributed current source. This problem is addressed by means of a Green's function technique akin to Shockley's impedance field method. To enable the efficient numerical evaluation of the Green's function within the framework of a discretized physical model, interreciprocity concepts, based on the introduction of an adjoint device, are exploited. Examples of implementation are discussed, relevant to the sensitivity and noise analysis of GaAs MESFET's.
\end{abstract}

\section{INTRODUCTION}

$\mathrm{T}$ HE IMPRESSIVE development of CAD tools for semiconductor device design based on physical models which has taken place over the last ten years only marginally has touched two important areas of device performance evaluation: sensitivity and noise simulation.

Although the concept of sensitivity analysis as a tool for device optimization had already been suggested in [1], the only method for device sensitivity analysis proposed so far to our knowledge is the technique for evaluating static small-change device sensitivities with respect to uniform doping and geometry variations presented in [2] and implemented in the HFIELDS simulator [3]. No attempt has been made to address other topics, such as ac sensitivity (i.e., the sensitivity of small-signal parameters) or the statistical sensitivity of the device with respect to random parameter variations [4].

Concerning noise analysis, classical analysis techniques, such as the impedance field method described by Shockley, Copeland, and James [5] and its extensions and generalizations have been available for many years (for a review, see, e.g., [6], [7]). Although such techniques are

Manuscript received March 11, 1991; revised March 10, 1992. This paper was recommended by Associate Editor D. Scharfetter.

G. Ghione is with the Dipartimento di Elettronica, Politecnico di Torino, Turin, Italy.

F. Filicori is with the Facoltà di Ingegneria, Università di Ferrara, Ferrara, Italy.

IEEE Log Number 9201145. well suited for numerical implementation in two- or threedimensional geometries, only applications to one-dimensional structures [8] or to quasi-physical models of GaAs FET's have been presented so far (see [9] and references therein).

Despite their different purposes, small-change parametric sensitivity and the noise analysis of semiconductor devices have a common background: both analyses require obtaining the small-signal response of the device to an equivalent distributed current source impressed either into the volume of the device or into its surface. Since the small-signal device model is linear, the response to an arbitrary impressed harmonic current density can be expressed in terms of the response to a spatially impulsive dipole source, also called the Green's function of the problem [10] and a superposition integral over the device volume. Moreover, linearity allows the device response to be uniquely expressed, for instance, in terms of the open-circuit voltages induced on the device ports. This choice justifies the name of vector impedance field $\mathbf{Z}$ originally assigned in [5] to the open-circuit voltage response to a spatially impulsive dipole source. It is worth stressing that the response to a dipole source, which is primary in noise and sensitivity device analysis, can, however, be derived from the response to a spatially impulsive scalar current source (i.e., the scalar impedance field $Z$ [5] ).

In sensitivity analysis, the source term arises from linear perturbation of the physical model as a dc (ac) current density for the static (small-signal) case, so that the smallsignal response is directly related to the device sensitivity. In noise analysis, the distributed current source models microscopic random fluctuations of the current density with respect to its average value, and the purpose of the analysis is to relate the power and correlation spectrum of the microscopic fluctuations (see, e.g. , [11], [5], [12] and references therein) to the power and correlation spectra of the voltage or current fluctuations induced into the external circuit connected to the device.

In order to formulate the problem into a more familiar form, it will be helpful to recall that the equations of the discretized device model can be interpreted in terms of an equivalent electrical network (cf. e.g., [13] and references therein). Therefore, the analysis of device sensitivity and noise can be more easily understood by consider- 
ing the well-known problem of evaluating the sensitivity and noise of an electrical network [14], [15]. Also in the latter case, sensitivity and noise can be evaluated from the linear response of the network to current sources related, respectively, to parameter variations or to the noise behavior of the individual components. The network analogy also offers a straightforward interpretation of the scalar impedance field. In fact, if the device model is discretized on a $N$-node mesh through a suitable numerical scheme, the discretized scalar impedance field $Z_{i j}$ is just the transimpedance between an internal node $j$ and the electrode $i$. Thus the potential induced by a distribution of current generators can be simply evaluated by superposition, which is a discrete form of the spatial superposition integral appearing in a Green's function formulation.

From a computational standpoint, the evaluation of the discretized scalar impedance field within the framework of a frequency-domain small-signal numerical device simulator [16], is a straightforward but computationally intensive task. In fact, it is necessary to repeatedly perform a device analysis while placing a single current source in each of the $N$ internal nodes, with a computational cost amounting to the solution of $N\left(N \approx 10^{3}-\right.$ $10^{4}$ ) linear systems of dimension $\approx N$, or, at least, to one LU factorization and $N$ backsubstitutions for each frequency. While a Green's function formulation cannot be avoided in noise analysis, where the total available noise power at the device terminals is obtained by power summation of spatially uncorrelated internal noise sources, ${ }^{1}$ sensitivity analysis does not strictly require a Green's function approach. This happens because the small-change device sensitivity with respect to a parameter can be described as the response to a well-defined current source distribution proportional to the parameter variation, and can, therefore, be obtained by globally solving a single current-driven problem. Nevertheless, in the Green's function approach the device sensitivity with respect to a parameter is expressed as a volume integral (or, in discretized form, as a node summation), whose integrand can be interpreted as a distributed parametric sensitivity. The knowledge of the spatial behavior of the sensitivity clearly allows deeper insight into which regions of the device display high or low sensitivity with respect to some parameter and is, therefore, a valuable design tool.

A computationally efficient technique for the noise and sensitivity analysis of semiconductor devices, which drastically reduces the computational cost of evaluating the Green's function, can be derived from the principle of the adjoint system approach [14], [15], [17] proposed during the late 1960 's for the noise and sensitivity analysis of large electrical networks. The adjoint approach avoids the repeated evaluation of the network response to a current generator placed in turn in each of the internal nodes by exploiting the fundamental property of direct-

\footnotetext{
'The formulation can be extended so as to deal with spatially correlated noise sources; see Section II-C.
}

adjoint linear system pairs, i.e., their being mutually reciprocal, or interreciprocal. The idea is trivial in a reciprocal network where the direct and adjoint system coincide; thus the transimpedance $Z_{i j}$ where $i$ is any of the internal nodes and $j$ is an external port, is equal to $Z_{j i}$, and can, therefore, be evaluated from the solution of one linear problem, i.e., by connecting a current generator to port $j$ and computing the resulting internal potentials. For any nonreciprocal linear network simple rules ultimately derived from the application of Tellegen's theorem enable the definition of an adjoint network such that the direct and adjoint pair are interreciprocal [14].

The purpose of the present paper is twofold. First, a unified treatment of the sensitivity and noise analysis of semiconductor devices is provided in which, for the first time to our knowledge, sensitivity analysis is reduced to evaluating the device response to a set of equivalent, distributed perturbation current density sources. Second, the idea of exploiting the interreciprocity properties of an adjoint system is extended to the numerical analysis of sensitivity and noise of semiconductor devices.

A drift-diffusion majority-carrier model will be assumed as the basis of the discussion, and application of the adjoint method to GaAs MESFET's will be considered as a case study. Nevertheless, the adjoint approach can in principle be extended to two-carrier or nonstationary (hydrodynamic) models. In order to derive the discretized adjoint device from the direct one by formal application of network rules [14], the small-signal majority carrier model is expressed in an admittance-like form, already proposed by the authors for computational expediency [18], [19]. The extension of the adjoint approach to two-carrier models which is currently under development requires on the other hand the use of more general forms of the interreciprocity theorem, in which the simple formal network analogy is no longer helpful.

The paper is structured as follows: Section II presents a unified discussion of sensitivity and noise analysis in terms of the small-signal response of the device to a harmonic, distributed current source. Section III introduces the adjoint technique, while its numerical implementation is described in Section IV. Finally, Section V is devoted to the discussion of some results concerning examples of sensitivity and noise analysis of GaAs MESFET's.

\section{A Unified Approach to Sensitivity and Noise Analysis of Semiconductor Devices}

From a physics-based standpoint, parametric sensitivity and noise analysis apparently have very little in cornmon. In sensitivity analysis, the effect of parameter variations on the device response is investigated; in the particularly important case where the parameter change is small, the device behaves as a linear system (small-change sensitivity analysis). In noise analysis, a deterministic device model is considered involving average values of microscopic quantities, such as the charge density and electric potential. Then microscopic fluctuations are 
superimposed, which can be modeled as random, distributed, harmonic current sources, and the response to such sources is investigated [5]. Since current fluctuations have small amplitude, the device again behaves as a linear system. Therefore, both small-change sensitivity and noise analysis involve the evaluation of the small-signal response of the device to a distributed source term related either to parameter variations or to microscopic current or charge fluctuations. However, while noise in the external circuit connected to the device naturally appears as the response to microscopic current fluctuations occurring with the device, the interpretation of the static and smallsignal sensitivity as the response to distributed dc or ac current sources is less straightforward. The present section is aimed at giving a formal background to the above ideas so as to establish a common framework for smallchange sensitivity and noise device analysis.

\section{A. The Physical Model}

Although most of the concepts introduced in the following sections can be readily applied to other, more complex, physical models, a majority-carrier drift-diffusion model will be assumed as the basis for the discussion. In large-signal operating conditions the model equations read

$$
\begin{aligned}
-q \frac{\partial n}{\partial t}+\nabla \cdot\left(J+J_{s}\right) & =0 \\
\epsilon \nabla^{2} \phi & =q\left(n-N_{D}^{+}\right)
\end{aligned}
$$

where $J=q[-n \mu \nabla \phi+D \nabla n]$ is the drift-diffusion current density and $J_{s}$ is an impressed distributed current source, which is zero under normal operating conditions and is introduced here for formal expediency, to be exploited later. In the above equations $n$ is the electron density, $\phi$ the electric potential, $\mu$ and $D$ the (field-dependent) electron mobility and diffusivity, $q$ the electron charge, $\epsilon$ the semiconductor permittivity, while $N_{D}^{+}$is the net equivalent ionized donor density; for the sake of simplicity, the ionization is assumed to be constant. The boundary conditions associated to the drift-diffusion system are well known, see, [20]. From the Poisson-continuity system, an equation can be derived in the potential only, which is useful to establish a direct analogy with the nodal formulation of an electrical network. On substituting in (1) the charge density derived from (2), the following expression is obtained:

$$
\begin{gathered}
-\epsilon \nabla^{2} \dot{\phi}+\nabla \cdot\left[-q N_{D}^{+} \mu \nabla \phi-\epsilon \mu \nabla^{2} \phi \nabla \phi+q D \nabla N_{D}^{+}\right. \\
\left.+\epsilon D \nabla \nabla^{2} \phi\right] \equiv \mathfrak{J}(\phi, \dot{\phi})=-\nabla \cdot J_{s}
\end{gathered}
$$

where $\dot{\phi}=\partial \phi / \partial t$. Similarly, the boundary conditions can be entirely expressed in terms of the unknown $\phi$ and its spatial derivatives; for the sake of brevity, these will be denoted as $\chi(\phi, s)=0$, where $\chi$ is a partial differential operator acting on the contour of the device and $s$ is a set of external sources applied at the device terminals.

\section{B. Sensitivity Analysis}

To carry out a formal sensitivity analysis, let us set the impressed term $\boldsymbol{J}_{s}$ to zero and make $\mathfrak{J}$ and $\chi$ explicitly dependent on a parameter set $p$, which can be either a single parameter, a discrete set of parameters, or a continuous function (e.g., the doping profile). Since from the standpoint of a numerically discretized problem the parameter set always has finite dimension, we shall unrestrictively consider $p$ to be a vector of dimension $N_{p}$. We have, therefore, $J(\phi, \dot{\phi}, p)=0$ with associated boundary conditions $\chi(\phi, s, p)=0$. Let us suppose we apply to the device a dc bias $s_{0}$ superimposed on small-amplitude timevarying ac generators $\hat{s}$, i.e., a small-signal excitation; at the same time, let the parameters undergo a small variation $\Delta p$ with respect to a reference value $p_{0}$ :

$$
\begin{aligned}
& s=s_{0}+\hat{s}(t) \\
& p=p_{0}+\Delta p .
\end{aligned}
$$

The resulting potential distribution will be written as

$$
\phi=\phi_{0}+\Delta \phi_{0}+\hat{\phi}(t)+\Delta \hat{\phi}(t)
$$

where $\phi_{0}$ is the dc response with nominal parameters, $\Delta \phi_{0}$ the dc perturbation caused by the parameter variation, $\hat{\phi}(t)$ the small-signal potential due to the time-varying part of the impressed generators $\hat{s}(t)$, i.e., the small-signal response and $\Delta \hat{\phi}(t)$ the variation of the latter due to the parameter variation. In the linear approximation, one clearly has $\Delta \phi_{0} \propto \Delta p, \hat{\phi}(t) \propto \hat{s}(t)$, while $\Delta \hat{\phi}(t) \propto \hat{s}(t) \Delta p$. If the expressions of (4), (5), and (6) are introduced into (3) and the operator and boundary conditions are expanded in Taylor series around the point $\left(s_{0}, \phi_{0}, p_{0}\right)$, the zeroth-order term yields the nominal (i.e., unperturbed) dc problem:

$$
\begin{array}{r}
3\left(\phi_{0}, 0, p_{0}\right)=0 \\
\chi\left(\phi_{0}, s_{0}, p_{0}\right)=0
\end{array}
$$

while first-order terms in $\Delta p$ yield the perturbed dc problem:

$$
\begin{aligned}
& \mathfrak{J}_{\phi} \Delta \phi_{0}=-\mathfrak{J}_{p} \Delta p \\
& \chi_{\phi} \Delta \phi_{0}=-\chi_{p} \Delta p
\end{aligned}
$$

where $J_{\alpha}$ stands for the gradient of $J$ with respect to $\alpha$, and all gradients are evaluated at the operating point $\left(\phi_{0}\right.$, $\left.s_{0}, p_{0}\right)$ and in de steady-state conditions $(\phi=0)$. Similarly, by taking the terms of first order in $\hat{s}$, one has the ac small-signal problem, which can be written in the frequency domain:

$$
\begin{aligned}
\mathcal{Y}(\omega) \hat{\phi}(\omega) & =0 \\
\chi_{\phi} \hat{\phi}(\omega) & =-\chi_{s} \hat{s}(\omega)
\end{aligned}
$$

where $\hat{\phi}(\omega)$ and $\hat{s}(\omega)$ are the Fourier transforms of the small-signal potential and impressed sources, respectively, and $\mathcal{Y}(\omega)=\mathfrak{J}_{\phi}+j \omega J_{\hat{\phi}}$ is the small-signal admittance operator arising from the linearization of the driftdiffusion model. Notice that in (9) $J_{\phi}=\mathcal{Y}(0)$. Finally, 
the second-order bilinear terms of the order $\hat{s} \Delta p$ yield the perturbation of the ac small-signal problem, which reads in the frequency domain:

$$
\begin{aligned}
\mathcal{Y}(\omega) \Delta \hat{\phi}(\omega) & =-\left[\mathcal{Y}_{\phi}(\omega) \Delta \phi_{0}+\mathcal{Y}_{p}(\omega) \Delta p\right] \hat{\phi}(\omega) \\
\chi_{\phi} \Delta \hat{\phi}(\omega) & =-\left[\chi_{\phi \phi} \Delta \phi_{0}+\chi_{\phi p} \Delta p\right] \hat{\phi}(\omega)
\end{aligned}
$$

where $\mathcal{Y}_{\alpha}$ is the gradient of $\mathcal{Y}$ with respect to $\alpha$. On introducing the inverse operator of $\mathcal{Y}, \mathcal{Z}$, the above equations can be rewritten in the following more explicit form:

$$
\begin{gathered}
\mathcal{Y}(\omega) \Delta \hat{\phi}(\omega)=-\left[\mathcal{Y}_{\phi}(\omega) \mathcal{Z}(0) \mathfrak{J}_{p}+\mathcal{Y}_{p}(\omega)\right] \hat{\phi}(\omega) \Delta p \\
\chi_{\phi} \Delta \hat{\phi}(\omega)=-\left[\chi_{\phi \phi} \mathcal{Z}(0) \mathfrak{J}_{p}+\chi_{\phi p}\right] \hat{\phi}(\omega) \Delta p .
\end{gathered}
$$

Inspection of the above systems suggests the following interpretations. It is clear from (9) that evaluating the static device sensitivity with respect to parameter variations reduces to computing the small-signal response to a dc distributed source term $\Delta J_{s}(r)$ such as:

$$
\nabla \cdot \Delta J_{s}=\mathfrak{J}_{p} \Delta p
$$

with boundary conditions given by (10). Comparison of (10) with (12) clearly shows that these boundary conditions are equivalent to a source term impressed on the device periphery. According to the type of problem considered, either the volume or the boundary term can vanish. ${ }^{2}$ Similarly, the ac sensitivity analysis amounts to evaluating the small-signal response to an ac distributed source term $\Delta \hat{\boldsymbol{J}}_{s}(\boldsymbol{r}, \omega)$ bilinearly related to the small-signal potential and to the parameter variation $\Delta p$, such as

$$
\nabla \cdot \Delta \hat{J}_{s}=\left[\mathcal{Y}_{\phi}(\omega) \mathcal{Z}(0) J_{p}+\mathcal{Y}_{p}(\omega)\right] \hat{\phi}(\omega) \Delta p
$$

with boundary conditions given by (16), again equivalent to an ac boundary source.

A final remark is suggested by comparison of (13) and (9). While for the dc sensitivity analysis the equivalent source term depends only on the results of the dc analysis with reference parameter values, the ac source terms also contain the variation of the dc potential distribution, $\Delta \phi_{0}$. Therefore, the ac sensitivity analysis always requires the solution of $N_{p}$ dc small-change sensitivity problems necessary in order to set up the $N_{p}$ different perturbation distributed ac current sources to be used in (13) and (14). Thus the ac sensitivity analysis is intrinsically more computationally intensive than the dc one, independent of the technique exploited for evaluating the device response to the source term.

Response to harmonic current sources-Green's Function Approach: It has already been shown that the dc and ac sensitivity analyses amount to evaluating the small-signal response to an equivalent sensitivity current density volume source (cf. (9), (13)), subject to a set $\chi$ of linear boundary conditions, which may include a sensitivity sur-

\footnotetext{
${ }^{2}$ For instance, the sensitivity to dimension variations not affecting the distributed parameters of the device can be formulated as already implied in [4], so as to lead to a boundary source term only.
}

face excitation (see (10), (14)). As already pointed out, either the volume or the surface excitation can vanish, according to which sensitivity parameter is considered. Finally, a surface excitation also appears in evaluating the nominal small-signal response of the device (see (12)). Since in linear operating conditions the superposition principle holds, the device response to surface and volume sensitivity sources can be separately evaluated so as to characterize the equivalent generators appearing at the device ports, while the nominal small-signal response can be uniquely derived from a linearly independent set of small-signal parameters. For the sake of simplicity, we shall concentrate mainly on the treatment of volume sources, which are also fundamental in noise analysis.

Let us generally indicate a harmonic impressed volume current density source as $\hat{J}_{s}(r, \omega)$, where $\boldsymbol{r}$ is the position vector, and the corresponding induced small signal potential as $\hat{\phi}(r, \omega)$, which is the solution to the linear problem:

$$
\mathcal{Y}(\omega) \hat{\phi}=-\nabla \cdot \hat{J}_{s}(\omega)
$$

where the operator $\mathcal{Y}$, already introduced in the last section, is, for the sake of brevity implicitly associated to the boundary conditions defined by the boundary operator $\chi$. It can be noted that the boundary condition set $\chi$ is now homogeneous, that is, it does not include any impressed surface generators. For the drift-diffusion model defined by (3), the explicit expression of (19) can be readily derived as

$$
\begin{aligned}
\nabla \cdot & {\left[\left(D_{0} \nabla+\mu_{0} \varepsilon_{0}\right) \epsilon \nabla^{2} \hat{\phi}-\left(q n_{0} \underline{\mu}+j \omega \epsilon\right) \nabla \hat{\phi}\right] } \\
= & \nabla \cdot \hat{J}_{s}(\omega)
\end{aligned}
$$

where $\mu_{0}$ and $D_{0}$ are the dc operating point electron mobility and diffusivity, $\varepsilon_{0}$ the dc electric field, $n_{0}$ the dc electron concentration, $\hat{\phi}(\omega)$ the Fourier transform of the small-signal electric potential and $\underline{\underline{\mu}}$ is the small-signal equivalent mobility tensor

$$
\underline{\underline{\mu}}=\mu_{0}\left\{\boldsymbol{I}+\left.\frac{\partial \log (\mu)}{\partial \boldsymbol{\varepsilon}}\right|_{0}\left[\frac{\left(\varepsilon_{0}+V_{T} \nabla \log \left(n_{0}\right)\right) \varepsilon_{0}}{\epsilon_{0}}\right]\right\}
$$

where $I$ is the identity tensor and $V_{T}$ the voltage equivalent of temperature.

The potential induced by the volume source $\hat{J}_{s}(\boldsymbol{r}, \omega)$, $\hat{\phi}(r)$, can be generally expressed in terms of the vector Green's function $Z$ and of a spatial superposition integral [10] as

$$
\hat{\phi}(r, \omega)=\int_{\Omega} Z\left(r, r^{\prime}, \omega\right) \cdot \hat{J}\left(r^{\prime}, \omega\right) d r^{\prime}
$$

As shown in [5] (see also [7]), $Z$ can be derived from a potential $Z$ as

$$
\boldsymbol{Z}\left(\boldsymbol{r}, \boldsymbol{r}^{\prime}, \omega\right)=\nabla_{\boldsymbol{r}^{\prime}} \mathbf{Z}\left(\boldsymbol{r}, \boldsymbol{r}^{\prime}, \omega\right) .
$$

$Z$ can, in turn, be interpreted [5] as a scalar Green's function, since the response to a harmonic scalar current source 


$$
\begin{gathered}
\hat{\imath}(\boldsymbol{r}, \omega)=-\nabla \cdot \hat{J}^{3} \text { can be expressed in terms of } Z \text { as } \\
\hat{\phi}(\boldsymbol{r}, \omega)=\int_{\Omega} Z\left(\boldsymbol{r}, \boldsymbol{r}^{\prime}, \omega\right) \hat{i}\left(\boldsymbol{r}^{\prime}, \omega\right) d \boldsymbol{r}^{\prime} .
\end{gathered}
$$

The treatment of an impressed surface current source is similar to the case of volume sources; only the volume integral of (22) is replaced by a surface integral.

For open-circuit boundary conditions, $Z\left(r_{i}, r^{\prime}, \omega\right)$, where $r_{i}$ denotes the electrode $i$, coincides with the vector impedance field originally introduced by Shockley [5] as the open-circuit potential $\hat{e}_{i} \equiv \hat{\phi}\left(r_{i}\right)$ induced on electrode $i$ by a unit dipole source $\hat{\boldsymbol{P}}$ impressed in $\boldsymbol{r}^{\prime}$ (or, equivalently, induced by a spatially impulsive current density $\hat{J}$ $\left.=\hat{\boldsymbol{P}} \delta\left(\boldsymbol{r}-\boldsymbol{r}^{\prime}\right)\right)$. Similarly, in the same conditions $\boldsymbol{Z}\left(\boldsymbol{r}_{\boldsymbol{i}}, \boldsymbol{r}^{\prime}\right.$, $\omega)$ coincides with Shockley's scalar impedance field, defined [5] as the open-circuit voltage induced on the electrode $i$ by a unit current source $\hat{I}$ impressed in $r^{\prime}$ (or, equivalently, induced by a spatially impulsive scalar current density $\left.\hat{\imath}=\hat{I} \delta\left(r-r^{\prime}\right)\right)$.

The meaning of the scalar and vector impedance fields for a device having $M$ contacts (plus one ground terminal) is shown in Fig. 1. Expressing the vector Green's function in terms of a scalar Green's function, i.e., of the device response to a scalar current source connected between the ground and a point of the device, clearly leads to computational advantages.

Green's Function Formulation of Sensitivity: On the basis of the previous discussion, the expression of device sensitivities as superposition integrals is straightforward. Device sensitivities include the dc sensitivities of external variables such as open-circuit voltages or short-circuit currents, and the ac sensitivities of small-signal parameters. Since the problem is linear, the small-change sensitivity of any set of parameters can be derived from a linearly independent set of sensitivities. With no loss of generality, we shall consider here as the relevant electrical variable the electrode potential $e_{i}$ and its open-circuit variations. Let us suppose that $N_{p}$ parameters $p_{1} \cdots p_{N_{p}}$, are varied; the impressed distributed current source term will be linearly related to the parameter variations. For the dc case:

$$
\begin{aligned}
\Delta e_{i} & =\sum_{k=1}^{N_{p}}\left(\int_{\Omega} \nabla_{\boldsymbol{r}^{\prime}} \mathbf{Z}\left(\boldsymbol{r}_{i}, \boldsymbol{r}^{\prime}, 0\right) \cdot \frac{\partial \Delta \boldsymbol{J}\left(\boldsymbol{r}^{\prime}\right)}{\partial p_{k}} d \boldsymbol{r}^{\prime}\right) \Delta p_{k} \\
& =\sum_{k=1}^{N_{p}}\left(\int_{\Omega} s_{p_{k}}^{e_{i}}\left(\boldsymbol{r}^{\prime}\right) d \boldsymbol{r}^{\prime}\right) \Delta p_{k} \\
& =\sum_{k=1}^{N_{p}} S_{p_{k}}^{e_{i}} \Delta p_{k}
\end{aligned}
$$

where $S_{p_{k}}^{e_{i}}(\boldsymbol{r})$. is the sensitivity of $e_{i}$ with respect to $p_{k}$, which can be expressed as the volume integral of a distributed sensitivity $s_{p}^{e_{i}}(\boldsymbol{r})$.

For the ac case, the sensitivity of the impedance matrix elements $z_{i j}$ can be directly expressed as the sensitivity of

\footnotetext{
${ }^{3}$ From the continuity equation, the term $-\nabla_{r^{\prime}} \cdot \hat{\boldsymbol{J}}\left(\boldsymbol{r}^{\prime}\right)$ can be interpreted as the equivalent scalar current density $\hat{\imath}\left(\boldsymbol{r}^{\prime}\right)=j \omega q \hat{n}\left(\boldsymbol{r}^{\prime}\right)$ impressed into $\boldsymbol{r}^{\prime}$.
}

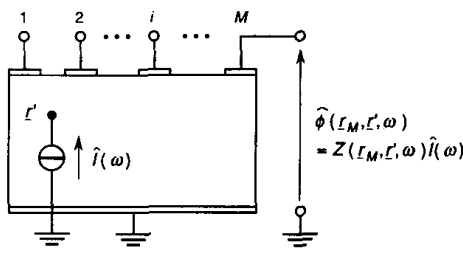

(a)

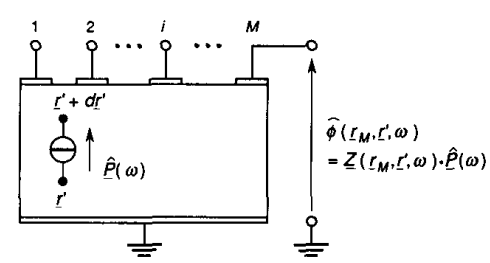

(b)

Fig. 1. Meaning of scalar (a) and vector (b) impedance fields for an $M$ terminal grounded device.

the open-circuit voltage $\hat{e}_{i}^{k}=z_{k i}$ induced on electrode $i$ by a unit current generator applied to the electrode $k$. If the distributed source corresponding to a set of parameter variations $\Delta p_{k}$ term is denoted as $\Delta \hat{\boldsymbol{J}}^{k}\left(\boldsymbol{r}^{\prime}\right)$ the variation $\Delta z_{k i}$ of the impedance matrix element $z_{k i}$ reads:

$$
\begin{aligned}
\Delta z_{k i} & =\sum_{k=1}^{N_{p}}\left(\int_{\Omega} \nabla_{\boldsymbol{r}^{\prime}} Z\left(\boldsymbol{r}_{i}, \boldsymbol{r}^{\prime}, \omega\right) \cdot \frac{\partial \Delta \hat{\boldsymbol{J}}^{k}\left(\boldsymbol{r}^{\prime}\right)}{\partial p_{k}} d \boldsymbol{r}^{\prime}\right) \Delta p_{k} \\
& =\sum_{k=1}^{N_{p}}\left(\int_{\Omega} s_{p_{k}}^{z k i}\left(\boldsymbol{r}^{\prime}\right) d \boldsymbol{r}^{\prime}\right) \Delta p_{k} \\
& =\sum_{k=1}^{N_{p}} S_{p_{k}}^{z k i} \Delta p_{k} .
\end{aligned}
$$

Both in the dc and the ac cases, the knowledge of the distributed sensitivity, rather than of only the overall sensitivity, can provide the device designer with valuable insight on how to properly optimize the device structure and technological process, since it allows the contribution of specific parts of the device to be singled out as critical. This information is altogether indispensable if one is interested in random parameter variations occurring in the device volume (e.g., random doping fluctuations or random geometry irregularities [4]); in this case, which is not discussed in detail here, the treatment is similar to that for noise analysis.

\section{Noise Analysis}

Noise in the circuit connected to a semiconductor device can be interpreted as the small-signal response to microscopic fluctuations $\delta J$ of the current density in the semiconductor. Random current fluctuations are modeled as a volume source term like the one introduced in (3) and denoted there as $J_{s}$; the device response is linear since the amplitude of fluctuations is small. For further details on the physical background, the reader can refer to [5], [11] and references therein. 
Compared to sensitivity analysis, noise analysis requires a further step, since the impressed current density $\delta J(r)$ is a space-dependent random process rather than a deterministic function. The aim of the analysis is therefore to obtain a second-order statistical characterization (i.e., the power and correlation spectra) of the equivalent external noise generators on the basis of the statistical properties of the microscopic current fluctuations. The treatment presented here closely follows the original formulation of [5] (see also [11], [7]) which is generalized so as to allow for spatially correlated microscopic noise sources.

Green's function formulation of noise analysis: Starting from (22), the power $(i=j)$ and correlation $(i \neq j)$ spectrum $\delta_{\delta e_{i} \delta e_{j}}$ of the induced potential fluctuations $\delta e_{i}\left(\delta e_{j}\right)$ on electrode $i(j)$ can be related to the correlation spectrum of the current fluctuations $\mathbf{S}_{\delta J\left(r_{1}\right) \delta J\left(r_{2}\right)}\left(r_{1}, \boldsymbol{r}_{2}, \omega\right)$ as: ${ }^{4}$

$$
\begin{aligned}
S_{\delta e_{i} \delta e_{j}}(\omega)= & \int_{\Omega} \int_{\Omega} \nabla_{r} Z\left(r_{i}, r_{1}, \omega\right) \cdot \mathbf{S}_{\delta J\left(r_{1}\right) \delta J\left(r_{2}\right)}\left(r_{1}, r_{2}, \omega\right) \\
& \cdot \nabla_{r} Z^{*}\left(r_{j}, r_{2}, \omega\right) d r_{1} d r_{2}
\end{aligned}
$$

where $\Omega$ is the device domain, and $*$ denotes complex conjugate. The correlation spectrum of the current density fluctuations is a matrix whose components are the Fourier transforms of the spatial correlation functions $R_{i j}$ defined in a rectangular reference system as $R_{i j}\left(r_{1}, r_{2}, \tau\right)=$ $E\left[\delta J_{i}\left(r_{1}\right) \delta J_{j}\left(r_{2}\right)\right]$, where $E[\bullet]$ denotes ensemble average.

Equation (27) can be considered as a generalization to three dimensions of the one-dimensional transmission line formulation of [22]. Since the correlation length of current fluctuations is of the order of the mean free path in the semiconductor, the microscopic sources can be assumed to be spatially uncorrelated in most practical situations. In this case

$$
\mathbf{S}_{\delta J\left(r_{1}\right) \delta J\left(r_{2}\right)}\left(r_{1}, r_{2}, \omega\right)=\mathbf{K}_{\delta J \delta J}\left(r_{1}, \omega\right) \delta\left(r_{1}-r_{2}\right)
$$

where $\mathbf{K}$ is usually referred to as local noise source [12]. for uncorrelated diffusion noise [5], [11] the local noise source takes the form:

$$
\mathbf{K}_{\delta J \delta J}(\boldsymbol{r}, \omega)=4 q^{2} \mathbf{D}_{0}(\boldsymbol{r}) n_{0}(\boldsymbol{r})
$$

where $\mathbf{D}_{0}$ is the diffusivity tensor and $n_{0}$ the electron density at the nominal dc operating point.

Assuming spatially uncorrelated sources in (27) finally leads to the classical impedance-field formula [5]:

$$
\begin{aligned}
\oint_{\delta e_{i} \delta e_{j}}(\omega)= & \int_{\Omega} \nabla_{r} Z\left(r_{i}, r, \omega\right) \cdot \mathbf{K}_{\delta J \delta J}(r, \omega) \\
& \cdot \nabla_{r} Z *\left(r_{j}, r, \omega\right) d r .
\end{aligned}
$$

\footnotetext{
${ }^{4} \mathrm{~A}$ rigorous proof based on the theory of distributed linear systems with random inputs (see [21]) is straigthforward but lengthy, and will not be given here. The result is often obtained in a heuristic way by taking (22), multiplying by its complex conjugate, taking the ensemble average, and exchanging integrals and averages.
}

\section{The Adjoint Approach}

In the previous sections, it has been shown that the device sensitivity and noise can be conveniently expressed through the scalar Green's function $Z$ (see (25), (26), (30)). To evaluate $Z$, a discretization scheme is introduced whereby the small-signal Poisson-continuity system, and, therefore, (20), is replaced by a discrete system on a mesh with $N$ nodes.

In this way, the continuous problem:

$$
\mathcal{Y} \hat{\phi}=-\nabla \cdot \hat{J}=\hat{\imath}(\boldsymbol{r})
$$

is turned into a discretized, admittance-like system of equations

$$
\mathbf{Y} \hat{\boldsymbol{\phi}}=\hat{I}
$$

where $\hat{\boldsymbol{\phi}}$ and $\hat{\boldsymbol{I}}$ are arrays of nodal potentials and node impressed currents, respectively, and $\mathbf{Y}$ is an admittance matrix whose explicit expression will be given in the next section.

Since the discretized scalar impedance field $Z_{i j}$ is the transimpedance between an internal node $j$ and an electrode node $i$, (i.e., $\hat{e}_{i}=Z_{i j} \hat{I}_{j}$ ), each element $Z_{i j}$ could be simply obtained from the solution of (32) with source term $\hat{I}=\left(0, \cdots, \hat{I}_{j}, \cdots, 0\right), j=1 \cdots N$, as $Z_{i j}=\hat{e}_{i} / \hat{I}_{j}$. The computational burden, however, would be fairly high, since for each frequency it would amount to the solution of $N\left(N \approx 10^{3}-10^{4}\right)$ linear equation systems of dimensions $\approx N \times N$, having the same coefficient matrix and $N$ different forcing terms; this involves only one LU factorization and $N$ back substitutions for each frequency. The same problem arising in the sensitivity and noise analysis of electrical networks was effectively solved through the so-called adjoint approach [15], [14]. The basic idea can be extended to device simulation as follows.

Let us start with the discretized system of (32) and introduce an adjoint system defined by the equation

$$
\mathbf{Y}^{\dagger} \hat{\boldsymbol{\phi}}^{\dagger}=\hat{\boldsymbol{I}}^{\dagger}
$$

where $\hat{\phi}$ and $\hat{\boldsymbol{I}}^{\dagger}$ are the adjoint nodal potential and current source, respectively, and $\mathrm{Y}^{\dagger}$ is the (heretofore unknown) admittance matrix of the discretized adjoint device. Consistently with the definition of adjoint network, we require that the adjoint system be interreciprocal to the direct one, meaning that $Z_{j i}^{\dagger}(\omega)=Z_{i j}(\omega)$, where $Z_{i j}^{\dagger}$ is the discretized scalar adjoint impedance field, subject to a set $\chi^{\dagger}$ of adjoint boundary conditions. Interreciprocity implies that the impedance field can be evaluated by just one analysis performed on the adjoint system of (33), i.e., by impressing a current $\hat{I}_{i}^{\dagger}$ into the electrode node $i$ and evaluating the adjoint potential distribution $\hat{\phi}_{j}^{\dagger}, j=1, \cdots, N$. In fact one has

$$
Z_{i j}=Z_{j i}^{\dagger}=\hat{\phi}_{j}^{\dagger} / \hat{I}_{i}^{\dagger}
$$

so that only one solution of the adjoint system is needed to evaluate $\hat{\phi}^{\dagger}$. The direct-adjoint current-driven pair is shown in Fig. 2. In the direct problem, a unit current source is impressed into a discretization node $j$, and the open-circuit potential induced on the electrode $i$ is eval- 


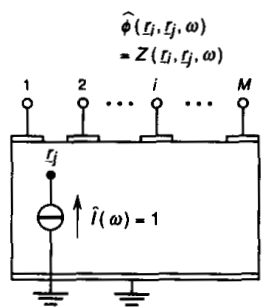

(a)

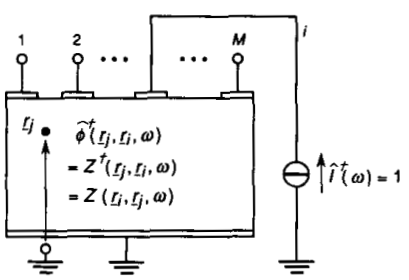

(b)

Fig. 2. (a) Current-driven direct device. (b) Adjoint device.

uated, which is equal to $Z_{i j}$. In the adjoint problem, a unit current source is impressed into the electrode $i$ and the resulting adjoint potential distribution $Z_{j i}^{\dagger}=Z_{i j}$ is obtained, thereby yielding the whole scalar impedance field with a single computation.

The identification of the adjoint device is more involved than that of the adjoint network, since a network is an intrinsically discrete system while a device is a continuous one, discretization being the result of a numerical approximation. The derivation of the discrete adjoint problem could therefore be performed either directly on the discretized device equations, or by first obtaining a continuous adjoint system, and then deriving the discretized adjoint therefrom.

In the first approach, the analogy between the discretized problem and the nodal formulation of an electrical network suggests the use of general rules [15], [17] for the identification of the adjoint system, thereby leading to the simple result $\mathbf{Y}^{\dagger}(\omega)=\mathbf{Y}^{T}(\omega)$, where $T$ stands for transpose; interreciprocity between the direct and adjoint system readily derives from Tellegen's theorem applied to the direct-adjoint pair [15].

In the second approach, standard techniques can be exploited to derive the adjoint equations and boundary conditions (see, e.g., [10]); interreciprocity directly derives from the classical definition of adjoint operator (for a more detailed discussion, see [23]).

From a computational standpoint, the first approach is clearly more convenient, not only because the discretization of the adjoint continuous problem is avoided, but also because in this way consistency with the direct discretized problem is guaranteed.

IV. IMPLEMENTATION OF THE ADJOINT APPROACH

The implementation of the adjoint approach has been carried out within the framework of a majority-carrier drift-diffusion device simulator based on a finite-boxes
Scharfetter-Gummel discretization scheme on an $N$ node grid [20]. The small-signal model is obtained by directly linearing around an operating point the large-signal timedomain system and by transforming the resulting linear time-domain system into the frequency domain. For further details on the present model the reader can refer to [18], [19].

The implementation is based on the following steps. First, the discretized direct small-signal frequency-domain current-driven problem is derived in admittance-like form [16]. Second, the small-signal parameters of the direct device are obtained. Finally, the discretized adjoint model is derived from the direct one.

To handle the discretized problem, we introduce the following notation. Let us define as free nodes those discretization nodes which do not lie on contacts, and as contact nodes those nodes which lie on contacts. Quantities relative to free nodes will be denoted by a subscript $f$, to contact nodes by a subscript $c$. Taking into account that the discretization admits $N_{f}$ free nodes and $N_{c}$ contact nodes $\left(N_{f}+N_{c}=N\right)$, all arrays of nodal values $\boldsymbol{x}$ can be partitioned in two subarrays: $\boldsymbol{x}=\left[\boldsymbol{x}_{f}, \boldsymbol{x}_{\boldsymbol{c}}\right]$, and similarly all $N \times N$ matrices $\mathbf{X}$ can be partitioned into four submatrices, as follows:

$$
\mathbf{X}=\left[\begin{array}{ll}
\mathbf{X}_{f f} & \mathbf{X}_{f c} \\
\mathbf{X}_{c f} & \mathbf{X}_{c c}
\end{array}\right]
$$

where $\mathbf{X}_{f f}$ and $\mathbf{X}_{c c}$ are square matrices of dimension $N_{f} \times$ $N_{f}$ and $N_{c} \times N_{c}$, respectively, while $\mathbf{X}_{f c}$ and $\mathbf{X}_{c f}$ are rectangular matrices of dimension $N_{f} \times N_{c}$ and $N_{c} \times N_{f}$, respectively.

Nodal formulation of the small-signal model: It can be readily shown [16], [18], [19] that the frequency-domain discretized small-signal system can be written in the form

$$
\left[\begin{array}{cc}
\mathbf{E} & j \omega \mathbf{B}+\mathbf{C} \\
\epsilon \mathbf{A} & q \mathbf{B}
\end{array}\right]\left[\begin{array}{l}
\hat{\boldsymbol{\phi}}(\omega) \\
\hat{n}(\omega)
\end{array}\right]=\frac{1}{q}\left[\begin{array}{l}
\hat{I} \\
0
\end{array}\right]
$$

where $\mathbf{B}$ is a diagonal area weight matrix, $\mathbf{A}$ the rigidity matrix of the Poisson equation, and $\mathbf{C}$ and $\mathbf{E}$ derive from the linearization of the continuity equation discretized according to the Scharfetter-Gummel scheme with respect to electron density and potential, respectively. For a detailed expression of these $N \times N$ matrices see [18], [19]. The array $\hat{\phi}(\omega)$ and $\hat{\boldsymbol{n}}(\omega)$ are the Fourier components of the electric potential and electron density at the angular frequency $\omega$, while $\hat{\boldsymbol{I}}$ is the array of current generators impressed into discretization nodes; finally, 0 is a $N \times 1$ null array. The previous arrays can be partitioned into a free and a contact part as follows:

$$
\begin{aligned}
\hat{\boldsymbol{\phi}}(\omega) & =\left[\begin{array}{l}
\hat{\boldsymbol{\phi}}_{f}(\omega) \\
\hat{\boldsymbol{\phi}}_{c}(\omega)
\end{array}\right] ; \quad \hat{\boldsymbol{n}}(\omega)=\left[\begin{array}{l}
\hat{\boldsymbol{n}}_{c}(\omega) \\
\hat{\boldsymbol{n}}_{c}(\omega)
\end{array}\right] ; \\
\hat{\boldsymbol{I}}(\omega) & =\left[\begin{array}{l}
\hat{\boldsymbol{I}}_{f}(\omega) \\
\hat{\boldsymbol{I}}_{c}(\omega)
\end{array}\right] .
\end{aligned}
$$


While homogeneous Neumann boundary conditions are already implicit in the discretization, on the ohmic contacts $\hat{\boldsymbol{n}}_{c}$ is assigned according to the neutrality condition, whereas $\hat{\boldsymbol{n}}_{c}$ is approximately zero on Schottky contacts in reverse bias. Therefore $\hat{n}_{c}=\boldsymbol{O}_{c}$ always unless the sensitivity has to be computed with respect to doping variations, for which $\hat{n}_{c i}=\Delta N_{D}$ on ohmic contacts and $\hat{n}_{c i}=$ 0 on Schottky contacts. Concerning the potential, the only boundary condition is that all nodes belonging to the same electrode have the same potential. In this way, only one unknown potential remains for each contact; on the other hand, for each electrode the current distribution is to be determined, the only datum being the total current for each electrode; therefore the system of (36) is completely defined. We shall not discuss in further detail the treatment of the current-driven problem, since as shown later, its explicit solution as such is not needed.

To proceed with the analysis, let us introduce the following matrix partitioning:

$$
\mathbf{E}=\left[\begin{array}{ll}
\mathbf{E}_{f f} & \mathbf{E}_{f c} \\
\mathbf{E}_{c f} & \mathbf{E}_{c c}
\end{array}\right] ; \quad \mathbf{C}=\left[\begin{array}{ll}
\mathbf{C}_{f f} & \mathbf{C}_{f c} \\
\mathbf{C}_{c f} & \mathbf{C}_{c c}
\end{array}\right]
$$

and similarly, taking into account that $\mathrm{A}$ is symmetric and B diagonal:

$$
\mathbf{A}=\left[\begin{array}{cc}
\mathbf{A}_{f f} & \mathbf{A}_{f c} \\
\mathbf{A}_{f c}^{T} & \mathbf{A}_{c c}
\end{array}\right] ; \quad \mathbf{B}=\left[\begin{array}{ll}
\mathbf{B}_{f f} & \boldsymbol{0}_{f c} \\
\boldsymbol{0}_{f c}^{T} & \mathbf{B}_{c c}
\end{array}\right]
$$

where $\boldsymbol{\theta}_{f c}$ is a null matrix of dimension $N_{f} \times N_{c}$ and $T$ stands for transpose.

Through Poisson's equation, $\hat{\boldsymbol{n}}(\omega)$ can be expressed in terms of $\hat{\phi}(\omega)$. This allows the electron density to be eliminated in the continuity equation, thereby leading to a nodal formulation in $\hat{\phi}(\omega)$ alone. In order to correctly include the charge neutrality condition $\hat{\boldsymbol{n}}_{c}=\boldsymbol{\theta}_{c}$ into the nodal formulation, one must express $\hat{n}_{c}$ in terms of nodal potential values, through the Poisson equation. The neutrality condition, expressed in terms of potential, reads:

$$
\hat{\boldsymbol{n}}_{c}=\frac{\boldsymbol{\epsilon}}{q} \mathbf{B}_{c c}^{-1}\left(\mathbf{A}_{c f}^{T} \hat{\boldsymbol{\phi}}_{f}+\mathbf{A}_{c c} \hat{\boldsymbol{\phi}}_{c}\right)=\boldsymbol{0}_{c} .
$$

As already mentioned, the neutrality condition has to be properly modified for dc sensitivity analysis with respect to doping variations, thereby originating a boundary source term whose detailed treatment is omitted for the sake of brevity.

By taking (38) into account, the nodal formulation in terms of potential (compared with (32)) can be expressed as

$$
\left[\begin{array}{ll}
\mathbf{Y}_{f f} & \mathbf{Y}_{f c} \\
\mathbf{Y}_{c f} & \mathbf{Y}_{c c}
\end{array}\right]\left[\begin{array}{l}
\hat{\boldsymbol{\phi}}_{f} \\
\hat{\boldsymbol{\phi}}_{c}
\end{array}\right]=\left[\begin{array}{l}
\hat{\boldsymbol{I}}_{f} \\
\hat{\boldsymbol{I}}_{c}
\end{array}\right]
$$

where the submatrices into which the nodal admittance matrix $\boldsymbol{Y}$ is partitioned read

$$
\begin{aligned}
& \mathbf{Y}_{f f}=q \mathbf{E}_{f f}-j \omega \epsilon \mathbf{A}_{f f}-\epsilon \mathbf{C}_{f f} \mathbf{B}_{f f}^{-1} \mathbf{A}_{f f} \\
& \mathbf{Y}_{f c}=q \mathbf{E}_{f c}-j \omega \epsilon \mathbf{A}_{f c}-\epsilon \mathbf{C}_{f f} \mathbf{B}_{f f}^{-1} \mathbf{A}_{f c}
\end{aligned}
$$

$$
\begin{aligned}
& \mathbf{Y}_{c f}=q \mathbf{E}_{c f}-j \omega \epsilon \mathbf{A}_{f c}^{T}-\epsilon \mathbf{C}_{c f} \mathbf{B}_{f f}^{-1} \mathbf{A}_{f f} \\
& \mathbf{Y}_{c c}=q \mathbf{E}_{c c}-j \omega \epsilon \mathbf{A}_{c c}-\epsilon \mathbf{C}_{c f} \mathbf{B}_{f f}^{-1} \mathbf{A}_{f c} .
\end{aligned}
$$

It is worth noting that the neutrality condition has been imposed on the nodal formulation by explicitly modifying the nodal admittance matrix according to (38).

Evaluation of the Small-Signal Parameters: In order to derive the small-signal parameters of the device from the nodal formulation of (39), the first step is to set to zero all current generators applied to free nodes $\left(\hat{\boldsymbol{I}}_{f}=\boldsymbol{\theta}_{f}\right)$, thereby leaving the contact excitation only. Then computational expediency suggests the use of a voltage-driven problem rather than of a current-driven one. In a voltagedriven problem, the contact part of the nodal potential array is assigned, i.e., $\hat{\phi}_{c}=\hat{V}_{c}$, where $\hat{V}_{c}$ is the small-signal potential applied to the contact nodes. On the other hand $\hat{\boldsymbol{I}}_{c}$ is an unknown which can be derived from the solution of the system, thereby yielding the current distribution on the electrodes and, therefore, the total small-signal currents. Thus, a reduced nodal formulation can be derived in the free nodes only, which reads:

$$
\mathbf{Y}_{f f}(\omega) \hat{\boldsymbol{\phi}}_{f}(\omega)=-\mathbf{Y}_{f c}(\omega) \hat{V}_{c}(\omega)
$$

while the contact currents can be obtained, after solving (44), from (39) as

$$
\hat{\boldsymbol{I}}_{c}=\mathbf{Y}_{c f}(\omega) \hat{\boldsymbol{\phi}}_{f}(\omega)+\mathbf{Y}_{c c}(\omega) \hat{\boldsymbol{V}}_{c}(\omega) .
$$

Finally, the total small-signal current is derived by summing all elements of $\hat{I}_{c}$ relative to the same contact. In a device having $M$ electrodes and one ground contact, $M$ small-signal solutions to independent voltage excitations are required to evaluate the admittance matrix. The computational burden corresponds for each frequency to the factorization of the reduced nodal matrix $Y_{f f}$ and $M$ back substitutions, one for each independent excitation.

Derivation of the Adjoint Problem: Taking into account that the direct problem of (39) is in admittance form, analogy to network analysis immediately yields the admittance matrix of the adjoint device:

$$
\mathbf{Y}^{\dagger}=\mathbf{Y}^{T}
$$

Therefore, the discretized adjoint system simply becomes (compare with (33)):

$$
\left[\begin{array}{cc}
\mathbf{Y}_{f f}^{T} & \mathbf{Y}_{c f}^{T} \\
\mathbf{Y}_{f c}^{T} & \mathbf{Y}_{c c}^{T}
\end{array}\right]\left[\begin{array}{l}
\hat{\boldsymbol{\phi}}_{f}^{\dagger} \\
\hat{\boldsymbol{\phi}}_{c}^{\dagger}
\end{array}\right]=\left[\begin{array}{c}
\hat{\boldsymbol{I}}_{f}^{\dagger} \\
\hat{\boldsymbol{I}}_{c}^{\dagger}
\end{array}\right]
$$

where $\hat{\phi}_{f}^{\dagger}, \hat{\phi}_{c}^{\dagger}$ are the adjoint potential on the free and contact nodes, respectively, while $\boldsymbol{I}^{\dagger}$ is the adjoint current impressed into the nodes. According to the direct-adjoint interreciprocal pair of Fig. 2, one chould now set to zero all bulk adjoint currents $\left(\boldsymbol{I}_{f}^{\dagger}=\boldsymbol{O}_{f}\right)$ and evaluate the adjoint potential distribution excited by a unit current generator impressed into one of the electrodes while the others are open. Again, the implementation of current boundary conditions, which is slightly inconvenient from the standpoint of device simulation, can be avoided in favor of more straightforward voltage boundary contitions, since 
the linearity of the adjoint problem enables the currentdriven problem in Fig. 2(a) to be replaced by an equivalent problem wherein the device is driven by a set of voltage sources $\hat{V}_{k}^{\dagger}=z_{k i}^{\dagger}=z_{i k}$, where $\mathbf{z}^{\dagger}=\mathbf{z}^{T}$ is the impedance matrix of the adjoint device.

Thus, in the voltage-driven adjoint problem $\hat{\boldsymbol{\phi}}_{c}^{\dagger}=\boldsymbol{V}_{c}^{\dagger}$ where $\hat{V}_{c}^{\dagger}$ is the assigned adjoint potential on the electrodes corresponding to a unit current excitation on terminal $i$; therefore, the reduced nodal formulation for the free nodes follows as:

$$
\mathbf{Y}_{f f}^{T}(\omega) \hat{\boldsymbol{\phi}}_{f}^{\dagger}(\omega)=-\mathbf{Y}_{c f}^{T}(\omega) \hat{\boldsymbol{V}}_{c}^{\dagger}(\omega) .
$$

The solution of (48) finally yields the distribution of the adjoint potential and, thus, with the above voltage excitation, the impedance field. Notice that, from a computational standpoint, the adjoint system matrix $\mathbf{Y}_{f f}^{T}$ is just the transpose of the direct one, which has already been LU factorized to perform the small-signal analysis. Since the solution of the transpose system can be obtained by exchanging and transposing the $L$ and $U$ factors, the computational cost of evaluating the impedance field relative to one electrode only amounts to one back substitution.

Discretization of Sensitivities and Noise Spectra: On applying simple first-order quadrature formulas consistent with the finite-boxes discretization scheme, the following expressions can be obtained for the dc sensitivity:

$$
S_{p_{k}}^{e_{i}} \approx \sum_{j=1}^{N} \nabla Z_{i j}(0) \cdot \frac{\partial \Delta J_{s}\left(r_{j}\right)}{\partial p_{k}} \Omega_{j}
$$

where the summation is extended to all $N$ discretization nodes, and $\Omega_{j}$ is the area of the polygonal box surrounding each node; similar expressions apply for the ac sensitivity case. for noise analysis

$$
S_{\delta e_{i} \delta e_{j}}(\omega) \approx \sum_{k=1}^{B} \nabla Z_{i k}(\omega) \cdot K_{\delta J \delta J}\left(r_{k}, \omega\right) \cdot \nabla Z_{j k}^{* T}(\omega) \Omega_{k} .
$$

For both sensitivity and noise analysis the numerical evaluation of the gradient of the scalar impedance field can be avoided by careful use of the Gauss theorem on the control volume surrounding each node.

\section{A. Computational Advantages of the Adjoint Approach}

In this section, the comparative computational cost of the $\mathrm{dc}$ and ac sensitivity analysis and that of the noise analysis with respect to the small-signal analysis alone will be investigated. In order to give at least an approximate quantitative basis for the comparison, let us introduce the following parameters: $C_{\mathrm{LU}}$ as the computational cost of the LU factorization of an $N \times N$ sparse matrix; $C_{s}$ as the cost of a back substitution on an $N \times N$ sparse linear system; $C_{p}$ as the cost of a scalar product between two vectors of length $N$. All matrices have dimension $N$, where $N$ is the number of discretization nodes. Taking into account that for an $M$ terminal device the evaluation of the complete set of $M$ linearly independent impedance fields amounts to the solution of $M$ adjoint problems for each frequency, i.e., to $M$ back substitutions on the transposed system, and supposing that the ac analysis is performed on $N_{f}$ frequencies and the sensitivity analysis involves $N_{p}$ parameters on a device with $M$ ports, it is easy to show that, neglecting the computational cost of matrix assumbling, one has

$$
C_{s s} \approx N_{f}\left[C_{L U}+M C_{s}\right]
$$

for the cost of the small signal analysis ${ }^{5}$

$$
C_{N} \approx N_{f} M C_{s}
$$

for the cost of noise analysis, roughly amounting to evaluating a set of independent Green's function for each frequency, i.e., to $M$ solutions of the adjoint problem. The cost of a dc sensitivity analysis is found to be

$$
C_{\mathrm{DC}} \approx M C_{s}+N_{p} M C_{p}
$$

where the first term is related to the evaluation of the $\mathrm{dc}$ Green's function, while the second one refers to the computation of (49) to be repeated for all parameters (see (25)). While dc sensitivity analysis is fairly inexpensive, the ac sensitivity analysis can be rather intensive. To fix the ideas, let us propose evaluating the sensitivity of the impedance or admittance matrix. The cost would be

$$
C_{\mathrm{AC}} \approx N_{p} C_{s}+N_{f} M C_{s}+N_{p} N_{f} M^{2} C_{p}
$$

where the second and third terms are similar to those arising in dc sensitivity, while the first one corresponds to evaluating the dc sensitivity of the static distribution for each of the parameters involved. Since usually $C_{\mathrm{LU}} \gg$ $C_{s}>C_{p}$, dc sensitivity and noise analysis can be performed with negligible overhead with respect to the smallsignal analysis alone for any value of $N_{p}$. On the other hand, the ac sensitivity analysis can become computationally intensive whenever $N_{p}$ is large, for instance when $N_{p}$ $=N$, as in statistical sensitivity analysis with respect to random parameter variations.

A final remark concerns the computational expediency of the adjoint implementation of the Green's function approach in device sensitivity analysis. As already stated in Section I, the response to an assigned perturbation distributed source can be obtained by a direct method, i.e., by solving one linear system with a given forcing term. If the Green's function were evaluated without making use of the adjoint technique by solving $N$ linear systems, the direct solution would be faster than the Green's function approach whenever $N_{p}<N$. Nevertheless, the adjoint approach reduces the computational cost of evaluating the Green's function to just one back substitution, thereby making it convenient with respect to the direct technique even for $N_{p}=1$.

\section{EXAMPLES}

This section discusses a few examples of the techniques presented in the paper. For sensitivity analysis, the simple but practically important case of dc sensitivity with re-

\footnotetext{
${ }^{5}$ As a reference the direct technique is assumed, rather than the iterative one described in [16].
} 


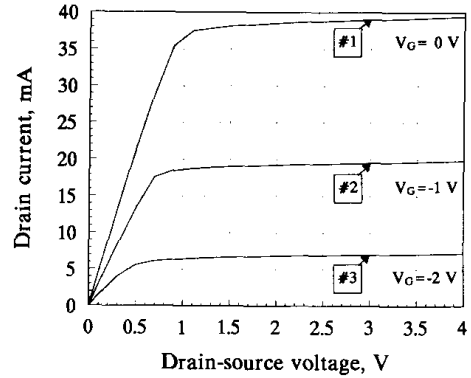

Fig. 3. Simulated dc characteristics of epitaxial $1-\mu \mathrm{m}$ MESFET. The operating points are: $\# 1, V_{\mathrm{GS}}=0 \mathrm{~V}, V_{\mathrm{DS}}=3 \mathrm{~V} ; \# 2, V_{\mathrm{GS}}=-1 \mathrm{~V}, V_{\mathrm{DS}}=$ $3 \mathrm{~V} ; \# 3, V_{\mathrm{GS}}=-2 \mathrm{~V}, V_{\mathrm{DS}}=3 \mathrm{~V}$.

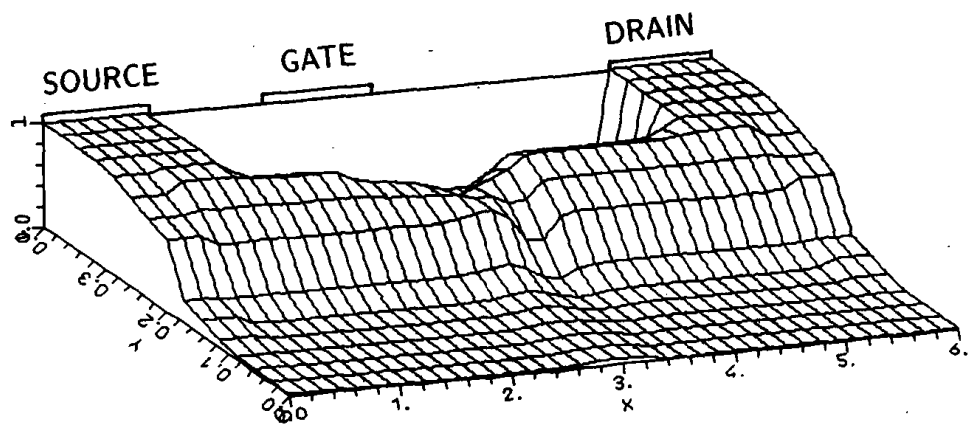

(a)

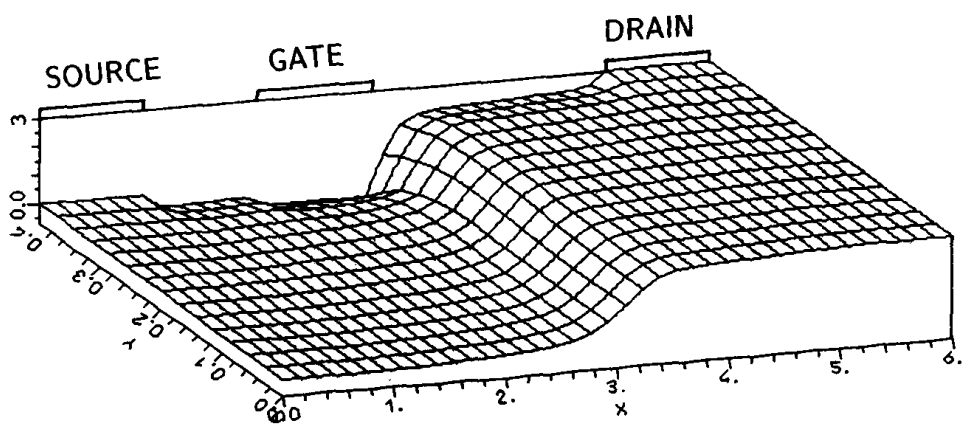

(b)

Fig. 4. dc charge and potential distributions for bias point \#1 of Fig. 3.

(a) Electron density, $10^{17} \mathrm{~cm}^{-3}$ units. (b) Electric potential, V.

spect to doping variations will be addressed in detail, with the aim of clarifying the general procedure presented in Section II-B with a specific example. For noise analysis, the purpose of the example shown is to demonstrate that the technique proposed leads to results which are in good agreement with other, simplified models (see [9]). A more complete discussion on the physical aspects of two-dimensional noise analysis is beyond the scope of this paper, and will be presented elsewhere. The sensitivity and noise analysis have been implemented within the framework of a two-dimensional drift-diffusion MESFET simulator derived from the one described in [18], [19]. All microscopic quantities are treated as invariant along the $z$ axis, and volume integrals are interpreted as surface integrals on a per unit length basis.
As a case study, an epitaxial 1- $\mu \mathrm{m}$ MESFET, with 300$\mu \mathrm{m}$ gate periphery, active layer thickness $0.2 \mu \mathrm{m}$, epilayer doping $N_{D}=10^{17} \mathrm{~cm}^{-3}$ and semi-insulating buffer layer has been considered. In all the computations shown, a nonuniform triangular mesh of about 1800 nodes was used. The dc curves are shown in Fig. 3; small signal and noise analysis was performed for $f=0, \cdots, 14 \mathrm{GHz}$ on the operating points marked in Fig. 3. The charge density and potential for the operating point \#1 are shown in Fig. 4.

A first result concerns the direct outcome of the adjoint technique, i.e., the frequency-dependent scalar impedance field $Z(\omega)$. Instead of $Z$, Fig. 5 shows (for the operating point \#1 and $f=4 \mathrm{GHz}$ ) a related function whose interpretation is easier: the short-circuit gate (Fig. 5(a), 


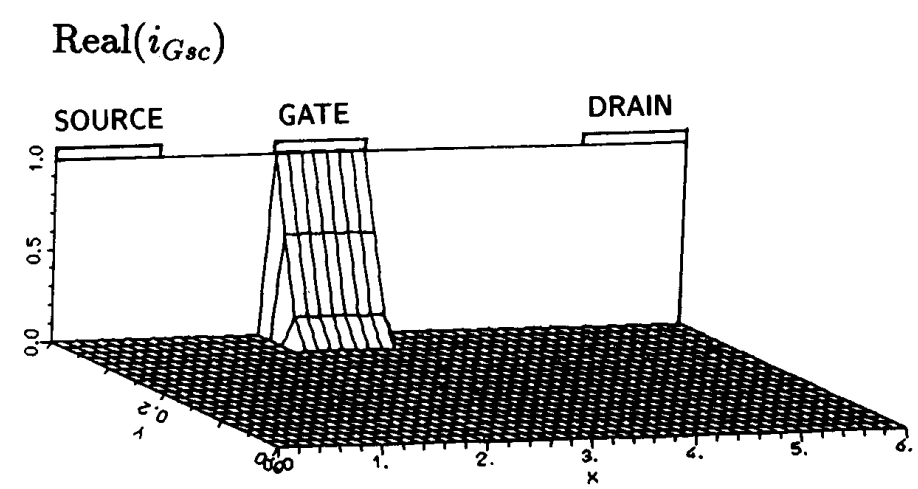

\section{$\operatorname{Imag}\left(i_{G s c}\right)$}

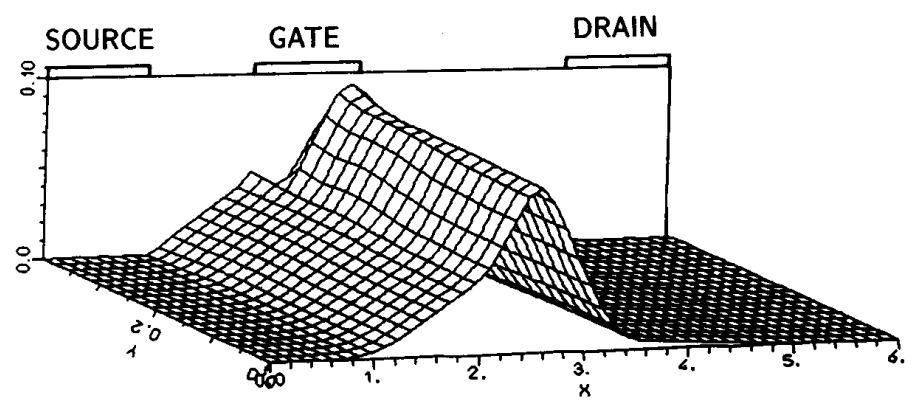

(a)

\section{$\operatorname{Real}\left(i_{D s c}\right)$}

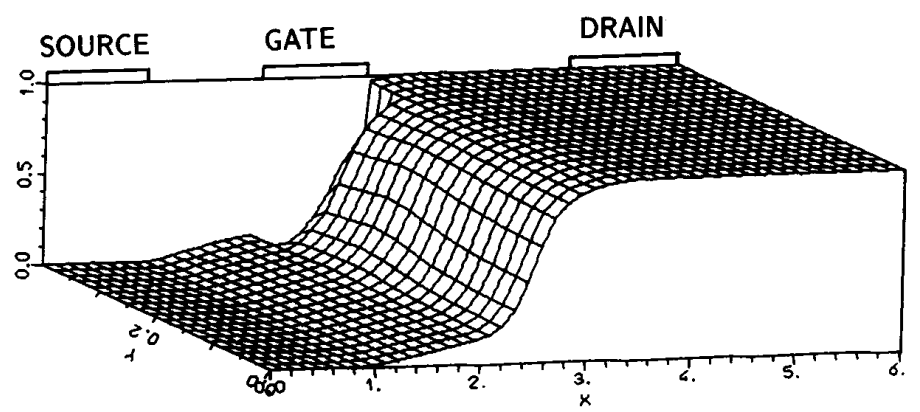

$\operatorname{Imag}\left(i_{D s c}\right)$

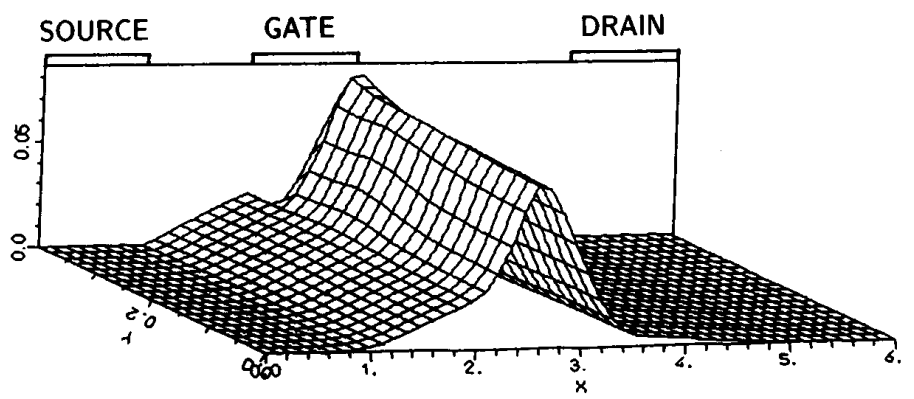

(b)

Fig. 5. (a) Small-signal short-circuit gate current for a unit harmonic current source injected into a point internal to the device. (b) Small-signal short-circuit drain current for a unit harmonic source injected into a point internal to the device. The frequency
is $f=4 \mathrm{GHz}$; the bias point is \#1 of Fig. 3 . 


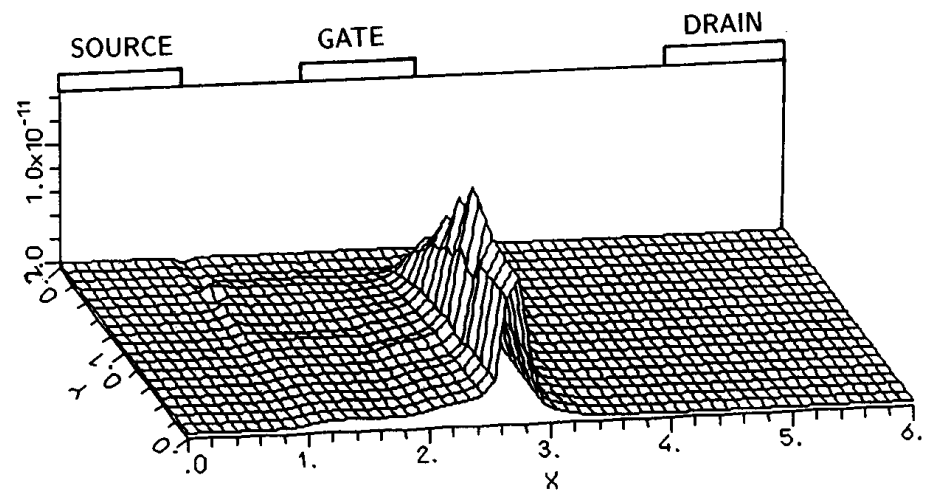

Fig. 6. Distributed sensitivity of the short-circuit drain current with respect to uniform variations of the doping level for the MESFET of Fig. 3, $\left(\mathrm{A} / \mathrm{m}^{-3}\right) \mathrm{m}^{-2}$ units; the bias point is \#1 of Fig. 3 .

(b)) and drain (Fig. 5(c), (d)) ac currents excited by a unit scalar current generator impressed into a point internal to the device. As already stressed, the set of small-signal short circuit currents can be easily related to the impedance field through the small-signal parameters of the device. Indeed, from a computational standpoint the shortcircuit currents are a more convenient choice than the impedance field in the analysis of insulated-gate FET's, since for such devices the impedance matrix becomes singular for $\omega \rightarrow 0$, whereas the admittance matrix is regular. Fig. 5(a), (b) clearly shows that the coupling between a current source impressed into the device and the gate short-circuit current is mainly capacitive; therefore, the induced gate current is in quadrature with the unit current source placed inside the device. The in-phase component of the gate short-circuit current is almost zero everywhere, apart from the gate itself, where it is equal to one. The abruptness of the jump shown in Fig. 5 is obviously proportional to the discretization density. The same remarks hold for the short-circuit induced drain current which is, however, mainly in-phase; the capacitive coupling increases with increasing frequency.

Sensitivity Analysis: As an example of sensitivity analysis, let us consider the static sensitivity of the device with respect to distributed variations of donot density $N_{D}$, $\Delta N_{D}(r)$. The doping variations cause an impressed charge density to appear in the Poisson equation, thereby leading to the equation in the potential only

$$
\begin{gathered}
\nabla \cdot\left[\left(D_{0} \nabla+\mu_{0} \varepsilon_{0}\right) \epsilon \nabla^{2}-q n_{0} \underline{\mu} \nabla\right] \Delta \phi_{0} \\
=q \nabla \cdot\left[D_{0} \nabla+\mu_{0} \varepsilon_{0}\right] \Delta N_{D}(r) .
\end{gathered}
$$

The associated boundary conditions also include the neutrality condition on ohmic contacts $\epsilon \nabla^{2} \Delta \phi_{0}=q \Delta N_{D}$. The open-circuit potential variation $\Delta \phi_{0}(r)$ caused by a doping variation $\Delta N_{D}(r)$ can be interpreted as the response to an impressed dc current density:

$$
\Delta J_{s}(r)=-q\left[D_{0} \nabla+\mu_{0} \varepsilon_{0}\right] \Delta N_{D}(r) .
$$

The doping variation $\Delta N_{D}(r)$ can in turn depend on other, more fundamental, process parameters. For in- stance, in the case of a single-implant profile, it would be

$$
\Delta N_{D}(r)=\frac{\partial N_{D}}{\partial D_{i}} \Delta D_{i}+\frac{\partial C_{D}}{\partial E_{i}} \Delta E_{i}
$$

where $D_{i}$ is the implanted dose and $E_{i}$ the implant energy. For a single epitaxial profile, as in the example considered here, a uniform doping variation of the epitaxial layer can be considered to be the sensitivity parameter. Therefore (56) simply yields $\Delta J_{s}(r)=-q \mu_{0} \varepsilon_{0} \Delta N_{D}$ and the dc sensitivity of the open-circuit voltage on electrode $i$ can be expressed as:

$$
\begin{aligned}
S_{N_{D}}^{e_{i}} & =\frac{\Delta e_{i}}{\Delta N_{D}}=-\int_{\Omega_{\mathrm{c}}} q \mu_{0} \nabla_{r} Z\left(r_{i}, r, 0\right) \cdot \varepsilon_{0} d r \\
& =\int_{\Omega_{\mathrm{c}}} s_{N_{D}}^{e_{i}}(r) d r
\end{aligned}
$$

where the integration domain $\Omega_{c}$ is the epitaxial layer.

For the reasons outlined above, the sensitivity of the short-circuit currents with respect to doping variations are a more significant parameter for MESFET's than that of the open-circuit voltages. Fig. 6 shows the distributed sensitivity $s_{N_{D}}^{i D}(r)$ of the dc drain current with respect to uniform doping variations in the epitaxial layer. Since $s_{N_{D}}^{i_{D}}(\boldsymbol{r})=0$ in the buffer layer, only the epitaxial layer has been simulated. As expected, the ohmic part of the device shows negligible distributed sensitivity, while maximum sensitivity can be found in the velocity-saturated part of the channel. The wriggles appearing in the distributed sensitivity near the source end of the devices are a numerical artifact due to discretization, since in that region the mesh density varies.

Noise Analysis: In the present results the only noise source considered is duffusion noise, with a scalar diffusion coefficient (see (29)). Diffusion noise is the prevailing noise mechanism at high frequency; nevertheless, other microscopic noise mechanisms, such as $1 / f$ noise or intervalley scattering noise, could be allowed for by properly modeling the microscopic noise source (see [12]). 


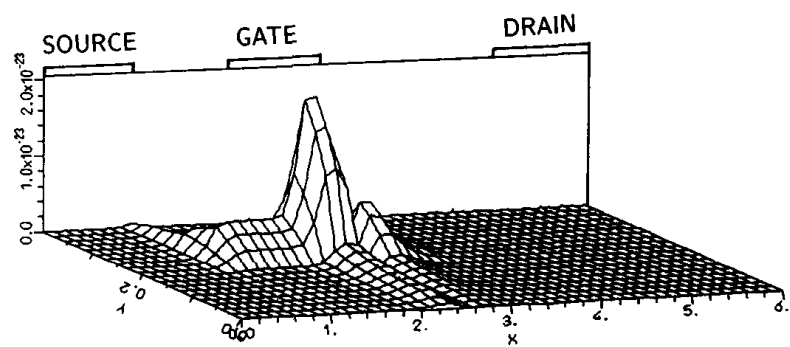

(a)

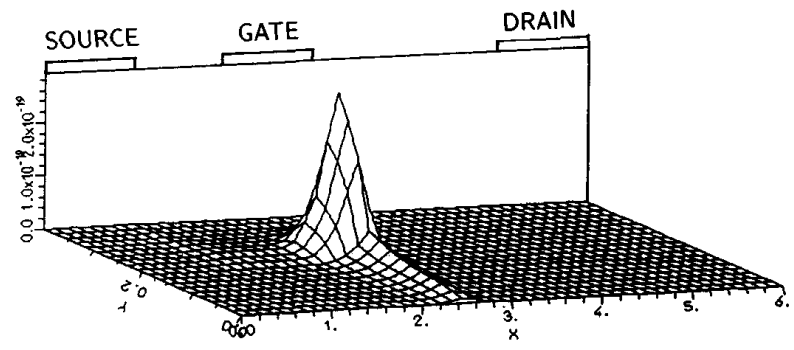

(b)

Fig. 7. Spatial density of power spectra of gate (a) and drain (b) shortcircuit current noise generators, $\mathrm{A}^{2} \mathrm{~Hz}^{-1} \mu \mathrm{m}^{-3}$ units; the bias point is $\# 1$ of Fig. 3.

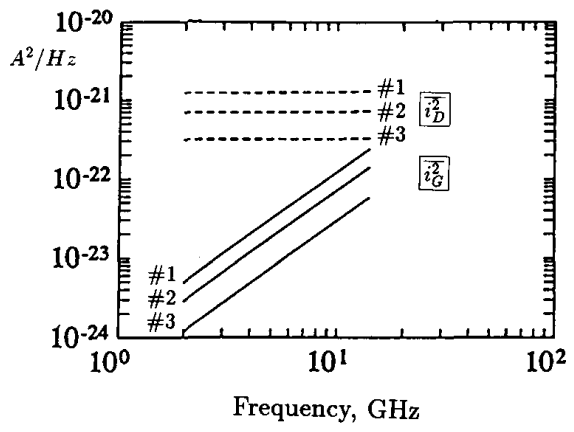

Fig. 8. Power spectra of gate and drain short-circuit noise generators, $\mathrm{A}^{2} / \mathrm{Hz}$ units, as a function of frequency; the bias points are those in Fig. 3 .

As in sensitivity analysis, the Green's function formulation allows the result (in this case the power and crosspower spectra of the electrical variables on interest) to be expressed in terms of the superposition of what could be defined the spatial density of the noise spectra. In the case where the noise spectra refer to open-circuit voltages, such a spatial noise density is nothing but the integrand of (30). The evaluation of the overall noise as the superposition of spatially uncorrelated noise contributions allows a direct physical interpretation of the noise orginating in different parts of the device.

As already recalled in the discussion concerning sensitivity analysis of FET's, in such devices short-circuit parameters or related quantities are more meaningful and easier to evaluate than open-circuit ones. Fig. 7 shows the

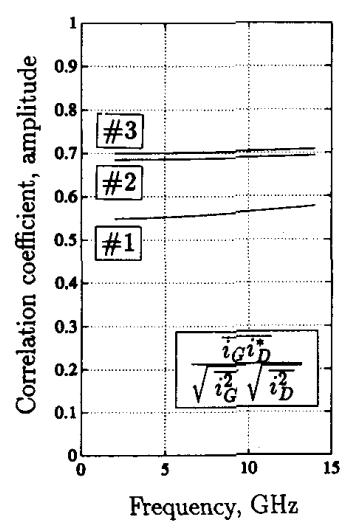

(a)

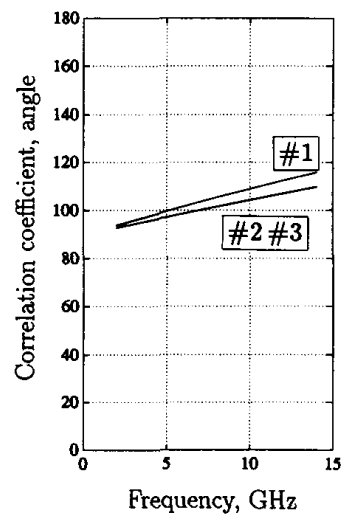

(b)
Fig. 9. (a) Amplitude degrees. (b) Phase degrees of the correlation spectrum of gate and drain short-circuit noise generators as a function of frequency; the bias points are those in Fig. 3 .

"spatial noise density" of the short-circuit gate and drain currents; in both cases the reference bandwidth is $1 \mathrm{~Hz}$. The major noise contributions are seen to originate from both the ohmic and saturated part of the channel, in agreement with the result from previous, analytical models (see [24]). Finally, Figs. 8 and 9 show the gate and drain noise short-circuit current spectral densities and the correlation coefficient, respectively, for all the operating points considered. The $\omega^{2}$ behavior of the gate generator is worth noting, as is that the correlation coefficient is almost imaginary at low frequency, in agreement with other analytical or one-dimensional noise models [24], [9]. The same sort of agreement is found for other parameters, such as the noise figure or the optimum generator impedance.

\section{Conclusions}

A unified treatment has been presented for the dc and ac sensitivity analysis and the noise analysis of semiconductor devices. Within this framework, an efficient technique has been proposed for the sensitivity and noise simulation of majority-carrier devices. The method, is based on the introduction of equivalent sensitivity and noise distributed current sources and on the concept of the adjoint device, can be easily implemented in a numerical device simulator, and requires little computational overhead with respect to the frequency-domain small-signal physical simulation. A few examples have been presented to illustrate the application of the method to the sensitivity and noise simulation of GaAs FET's.

\section{REFERENCES}

[1] J. Middleton and J. B. Waddell, "Sensitivity analysis and its application in the optimum design of semiconductor devices," in Proc. SISDEP, Swansea, U.K., 1984, pp. 286-297.

[2] A. Gnudi, P. Ciampolini, R. Guerrieri, M. Rudan, and G. Baccarani, "Sensitivity analysis for device design," in Proc, of ESSDERC 87, Bologna, Italy, Sept. 1987; pp. 551-554, also, IEEE Trans. Computer-Aided Design, vol. CAD-6, pp. 879-885, Sept. 1987.

[3] G. Baccarani, R. Guerrieri, P. Ciampolini, and M. Rudan, "HFIELDS: A highly-flexible 2-D semiconductor-device analysis 
program," in Proc. NASECODE IV, Dublin, Ireland, June 1985, pp. 3-12.

[4] G. De Mey, "Stochastic geometry effects in MOS transistors," IEEE J. Solid-State Circuits, vol. SC-20, pp. 865-870, Aug. 1985.

[5] W. Shockley, J. A. Copeland, and R. P. James, "The impedance field method of noise calculation in active semiconductor devices," in Quantum Theory of Atoms, Molecules and the Solid State, P. O Lowdin Editor. Orlando, FL: Academic, 1966, pp. 537-563.

[6] K. M. van Vliet and J. R. Fasset, "Fluctuations due to electronic transitions and transport in solids," in Fluctuation Phenomena in Sol$i d s$, R. E. Burgess, Editor. Orlando, FL: Academic, 1965, pp. 267354.

[7] K. M. van Vliet, A. Friedman, R. J. J. Zijlstra, A. Gisolf, and A. van der Ziel, "Noise in single injection diodes. I: A survey of methods," J. Appl. Phys., vol. 46, No. 4, pp. 1804-1816, Apr. 1975.

18] A. Moatadid, D. Gasquet, M. de Murcia, and G. P. Nougier, "Noise of GaAs Diodes," in Proc. ESSDERC 1988, Montpellier, France, Sept. 1988, pp. 583-n586.

[9] A. Cappy, "Noise modeling and measurement techniques,"' IEEE Trans. Microwave Theory Tech., vol. 36, pp. 1-10, Jan. 1988.

[10] B. Friedman, Principles and Techniques of Applied Mathematics. New York: Wiley, 1956.

[11] A. van der Ziel, Noise: Sources, Characterization, Measurement. Englewood Cliffs, NJ: Prentice-Hall, 1970.

[12] J. P. Nougier, "Noise and diffusion of hot carriers," in Physics of Nonlinear Transport in Semiconductors, D. K. Ferry, J. R. Barker, and C. Jacoboni Editors. New York: Plenum, 1980, pp. 415-465.

[13] C. T. Sah, "New integral representations of circuit models and elements for the circuit technique for semiconductor device analysis,' Solid-State Electron., vol. 30, no. 12, pp. 1277-1281, 1987.

[14] S. W. Director and R. A. Roher, "Automated network design-The frequency-domain case," IEEE Trans. Circuit Theory, vol. CT-16, pp. 330-337, Aug. 1969.

[15] R. A. Roher, L. Nagel, R. Meyer, and L. Weber, "Computationally efficient electronic-circuit noise calculation," IEEE J. Solid-State Circuits, vol. SC-6, pp. 204-212, Aug. 1971.

[16] S. E. Laux, "Techniques for small-signal analysis of semiconductor devices,' IEEE Trans. Electron Devices, vol. ED-32, pp. 2028-2037, Oct. 1985.

[17] F. H. Branin, "Network sensitivity and noise analysis simplified," IEEE Trans Circuit Theory, vol. CT-20, pp. 285-288, May 1973

[18] G. Ghione, C. Naldi, and F. Filicori, "Physical modeling of GaAs MESFET's in an integrated CAD environment: From device technology to microwave circuit performance,"' IEEE Trans. Microwave Theory and Tech., vol. 37, pp. 457-468, Mar. 1989.

[19] G. Ghione, C. Naldi, F. Filcori, M. Cipelletti, and G. Locatelli, "MESS-A Two-Dimensional Physical Device Simulator and its Application to the Development of C-band Power GaAs MESFET's," Alta Freq., vol. LVII, no. 7, Sept. 1988.

[20] S. Selberherr, Analysis and Simulation of Semiconductor Devices. New York: Springer-Verlag, 1984.

[21] A. Papoulis, Probability, Random Variables, and Stochastic Processes. Tokyo, Japan: McGraw-Hill Kogakusha, 1965.
[22] H. A. Haus, “'On noise analysis in bulk semiconductors,"' Solid State Electron., vol. 17, pp. 1075-1081, 1974

[23] G. Ghione, "A two-dimensional approach to the noise simulation of GaAs MESFET's," in Proc. ESSDERC 90, Nottingham, U.K., Sept. 1990, pp. 225-229.

[24] R. A. Pucel, H. A. Haus, and H. Statz, "Signal and noise properties of gallium arsenide microwave field-effect transistors," in Advances in Electronics and Electron Physics, vol. 38. New York: Academic, 1975 , pp. 195-265.

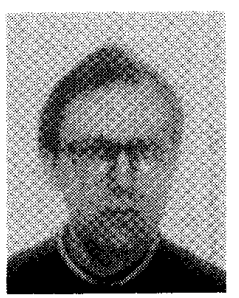

Giovanni Ghione (M'87) received the Laurea degree in electronic engineering from Politecnico di Torino, Turin, Italy, in 1981.

From 1983 to 1987 he was with the Department of Electronics of the same institution as a Researcher; from 1987 to 1990 he was with Politecnico di Milano as an Associate Professor. In 1990 he joined the University of Catania as a Full Professor in electronics. In 1991 he returned to the Politecnico di Torino as a Full Professor in solidstate devices. His current research interests concern the numerical modeling of passive and active components for hybrid and monolithic MIC's (coplanar and multiconductor lines, two-dimensional physical modelling of MESFET and HEMT devices, circuit-oriented nonlinear FET models) and the field of low- and high-frequency linear and nonlineaqr electromagnetics.

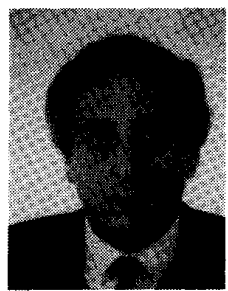

Fabio Filicori was born in Imola, Italy, in 1949. $\mathrm{He}$ received the Dr. Ing. degree in electronic engineering from the University of Bologna, Italy, in 1974 .

In 1974 the joined the Department of Electronics of the University of Bologna, first as an assistant researcher and, later, as an associate professor of applied electronics. In November 1990 he became full professor of applied electronics at the University of Perugia. In 1991, he joined the Faculty of Engineering of the University of Ferrara where he is a full professor, responsible for the curricula in electronic engineering. He has been teaching courses on computer-aided circuit design, electron devices and circuits, and power electronics. His main research activities are in the areas of computer-aided design techniques for nonlinear microwave circuits, electron device modeling, and electronic measurements.

Dr. Filicori is a member of Associazione Elettrotecnica ed Elettronica Italiana (AEI). 